\title{
O USO DO PNEUMOPERITÔNIO PROGRESSIVO NO PRÉ-OPERATÓRIO DAS HÉRNIAS VOLUMOSAS DA PAREDE ABDOMINAL
}

\author{
José Guilherme MINOSSI, Walmar Kerche de OLIVEIRA, Juan Carlos LLANOS, \\ Samuel Moraes IELO, Claudia Nishida HASIMOTO e Rodrigo Severo de Camargo PEREIRA
}

\begin{abstract}
RESUMO - Contexto - A correção das hérnias volumosas e dos grandes defeitos da parede abdominal constitui grande desafio da prática cirúrgica, em virtude das dificuldades técnicas e do alto índice de complicações respiratórias e cardiovasculares. Objetivos - Apresentar experiência com a indução do pneumoperitônio progressivo no pré-operatório do tratamento cirúrgico das hérnias volumosas da parede abdominal. Métodos - Estudo retrospectivo de seis pacientes que apresentavam hérnias volumosas da parede abdominal, e que foram operados após a instalação de um pneumoperitônio. O procedimento foi realizado através da colocação de um cateter na cavidade abdominal, na altura do hipocôndrio esquerdo, com insuflação de ar ambiente por período de 10 a 15 dias. Resultados - Dos seis pacientes operados, quatro eram do sexo feminino e dois do masculino. A idade mínima era de 40 e a máxima de 62 anos. A duração da hérnia variou de 5 a 40 anos. Quatro pacientes tinham hérnia incisional, um umbilical e outro inguinal. O tempo médio de pneumoperitônio foi de 11,6 dias. Não houve complicações relacionadas à instalação e manutenção do pneumoperitônio. Todas as hérnias foram corrigidas sem dificuldades técnicas. Utilizou-se a técnica de Lichtenstein para a correção da hérnia inguinal, a transposição peritônio-aponeurótica para uma das hérnias incisionais, sendo as demais corrigidas com uso de tela de polipropileno. Um óbito e uma infecção de parede foram observados no pós-operatório dessas cirurgias. Não houve recidivas registradas até o momento, num período de seguimento de 4 a 36 meses. Conclusão - O pneumoperitônio progressivo pré-operatório é um procedimento seguro e de fácil execução, pois facilita o procedimento cirúrgico e diminui as complicações respiratórias e cardiovasculares no pós-operatório. É indicado para doentes com hérnias que perderam domicílio na cavidade abdominal.

DESCRITORES - Pneumoperitônio artificial. Hérnia ventral. Hérnia inguinal.
\end{abstract}

\section{INTRODUÇÃO}

A correção das hérnias volumosas e dos grandes defeitos da parede abdominal constitui grande desafio da prática cirúrgica, em virtude das dificuldades técnicas e do alto índice de complicações locais e sistêmicas, como a recidiva e a infecção e, principalmente, as complicações respiratórias e cardiovasculares.

Em razão disso, muitos recursos têm sido utilizados no pré e no pós-operatório destas cirurgias, de modo a permitir a sutura dos tecidos sem tensão e também prevenir as complicações anteriormente citadas ${ }^{(6)}$.

A indução de pneumoperitônio progressivo pré-operatório visando o tratamento das hérnias muito grandes, com perda de domicílio na cavidade abdominal, foi introduzida na década de 1940 por GOÑI-MORENO(2). Posteriormente, HERSZAGE ${ }^{(3)}$, MARTINEZ ${ }^{(7)}$ e MAYAGOITIA et al ${ }^{(9)}$ promoveram interessantes modificações no método original.
O pneumoperitônio progressivo pré-operatório é recomendado para pacientes com hérnias volumosas, com grande quantidade de vísceras no saco herniário, cuja redução do conteúdo para o interior da cavidade peritonial pode levar o paciente a desenvolver síndrome compartimental abdominal no pós-operatório ${ }^{(11)}$.

A capacidade da cavidade abdominal varia de acordo com as alterações de volume do seu conteúdo. Na gravidez ou ascite, por exemplo, a parede abdominal distendese de forma gradual, aumentando consideravelmente o seu continente.

Nas hérnias volumosas, no entanto, parte do conteúdo abdominal se aloja no saco herniário, ocorrendo queda da pressão intra-abdominal e, consequentemente, adaptação da mesma a menor volume.

A queda da pressão intracavitária produz dificuldade no retorno venoso, vasodilatação e estase venosa abdominal, pélvica e dos membros inferiores. O diafragma também enfraquece, por não encontrar resistência à contração,

Trabalho realizado no Departamento de Cirurgia e Ortopedia, Disciplina de Gastroenterologia Cirúrgica da Faculdade de Medicina de Botucatu, Universidade Estadual Paulista - UNESP. Botucatu, SP.

Correspondência: Dr. José Guilherme Minossi - Praça Irmãos Ferreira, 171 - Centro - 18760-000 - Cerqueira César, SP. E-mails: jminossi@uol.com.br ; jminossi@fmb. unesp.br 
reduzindo a capacidade respiratória. Devido a dificuldade do retorno venoso e linfático, ocorre edema crônico no omento, mesentério e intestino. Essas alterações são toleradas porque se desenvolvem gradativamente.

A reposição das vísceras herniadas, nas grandes hérnias, às vezes é impossível ou transitoriamente possível, graças ao aprofundamento do plano anestésico ou à curarização. Passados esses efeitos, a desproporção entre o continente e o conteúdo se restabelece e o paciente entra em dificuldade respiratória, até mesmo asfixia, se não houver providências para sua prevenção.

Essas alterações agudas podem levar à atelectasia, insuficiência respiratória, hipovolemia, choque, trombofeblite e acidentes tromboembólicos, além da recidiva precoce ou mediata da hérnia ${ }^{(6)}$.

Nos doentes com hérnias com perda do domicílio, a indução de pneumoperitônio progressivo pré-operatório corrige alguns dos problemas citados, pois pode elevar novamente a pressão intra-abdominal de forma gradual e estabilizar a forma e a função diafragmática, melhorar a função ventilatória, distender os músculos da parede abdominal para aumentar o volume da cavidade. O pneumoperitônio também pode produzir a lise pneumática das aderências intestinais mais frouxas e melhorar a circulação portal, mesentérica e intestinal, para receber de volta as vísceras na cavidade abdominal ${ }^{(10)}$.

\section{Objetivo}

Apresentar a experiência do Serviço de Gastroenterologia Cirúrgica, do Hospital das Clínicas da Universidade Estadual Paulista - UNESP, Botucatu, SP, com a indução de pneumoperitônio progressivo pré-operatório para o tratamento cirúrgico das hérnias volumosas da parede abdominal. São discutidos, também, os aspectos técnicos, as indicações e as complicações do procedimento.

\section{MÉTODOS}

Os pacientes foram mantidos hospitalizados durante todo o período de duração das insuflações de ar ambiente. À internação, os mesmos assinaram um Termo de Consentimento Esclarecido, após explicações sobre as possíveis complicações do procedimento e da cirurgia.

Após período de jejum de 8 horas, o procedimento foi realizado na própria enfermaria ou no Centro Cirúrgico, local de escolha nos casos mais difíceis, como pacientes obesos ou com grandes hérnias, próximo ao local de punção.

Todas as punções foram realizadas no hipocôndrio esquerdo, sobre a linha hemiclavicular, junto ao rebordo costocondral.

Após a antissepsia da parede abdominal foi realizada a anestesia local e raramente a geral. Para a insuflação de ar, em pacientes não excessivamente obesos, um cateter deve ser inserido na cavidade abdominal através de uma punção com um "venocatch" (Figura 1). Por outro lado, nos pacientes muito obesos, o procedimento é realizado com uma agulha de Veress, de maneira idêntica à da laparoscopia (Figura 2). Nesses casos, o paciente deve ser levado ao Centro Cirúrgico, sendo insuflado gás carbônico até que o pneumoperitônio esteja instalado (aproximadamente 2 L). Em seguida, é realizada uma segunda

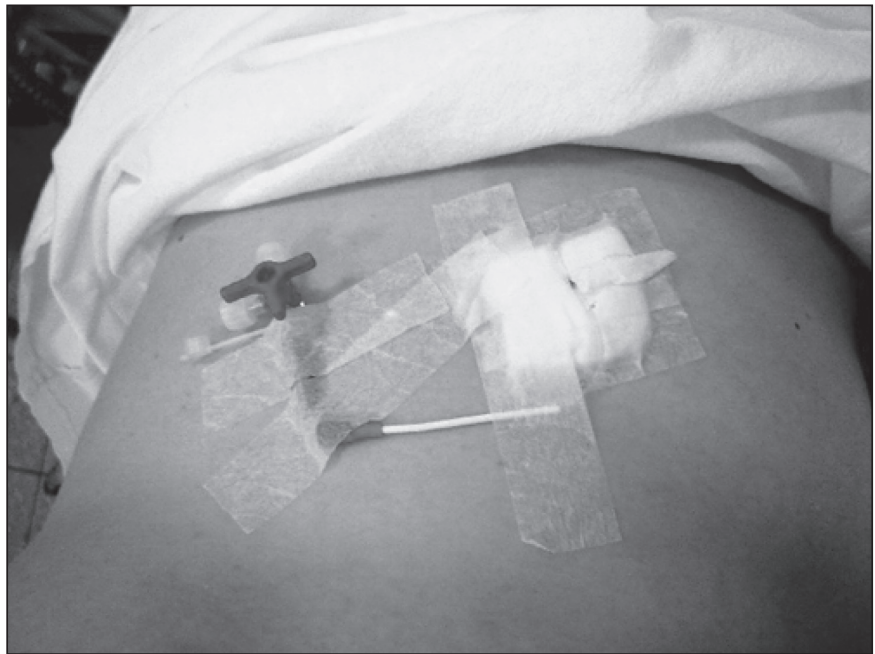

FIGURA 1. Paciente $n^{\circ} 3$. Pneumoperitônio instalado na enfermaria com venocatch. A figura mostra a retirada do cateter pouco antes da cirurgia, quando foi esvaziado o pneumoperitônio

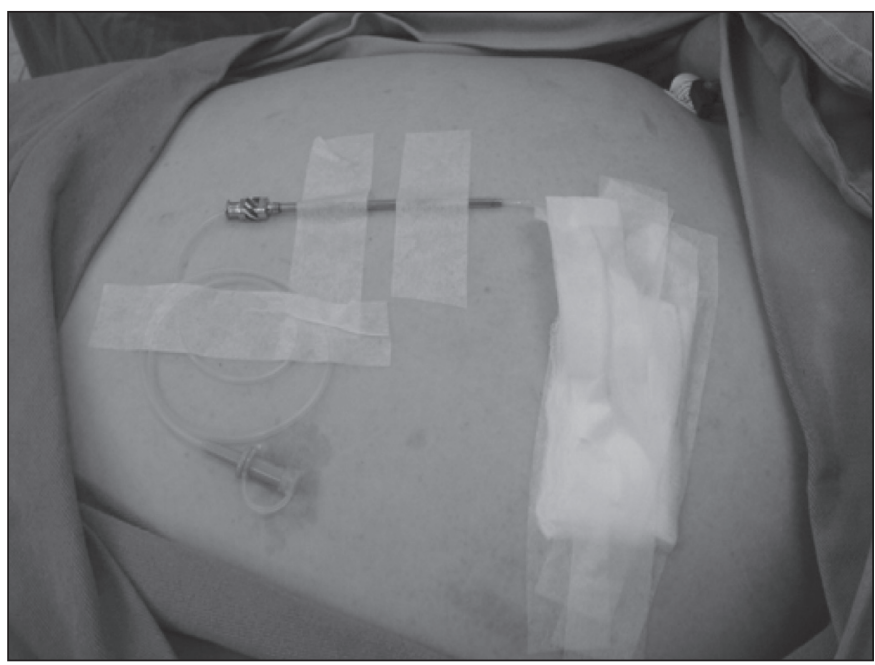

FIGURA 2. Paciente $n^{\circ}$ 2. Pneumoperitônio instalado no centro cirúrgico sob anestesia local. O cateter de Levine $n^{\circ} 6$ encontra-se fixado no hipocôndrio esquerdo. Observe a camisa da agulha de Coope, que fica até o final do procedimento ou pode ser retirada através do posicionamento de um cateter utilizando um fio guia

punção com agulha de Coope, pela qual é passada uma sonda de Levine $n^{\circ} 6$, que é fixada à pele. Uma torneira de três vias é conectada à extremidade do cateter.

Radiografia de cúpulas diafragmáticas deve ser realizada para confirmar a presença de pneumoperitônio. Nos casos em que o procedimento é realizado na enfermaria, a radiografia deve ser realizada após insuflação de $1 \mathrm{~L}$ de ar ambiente.

Constatada a presença de ar sob as cúpulas diafragmáticas, o paciente passa a receber insuflações diárias de ar ambiente em quantidades que variam de 1 a $2 \mathrm{~L}$ ao dia, por período de 10 a 15 dias. A tolerância ao pneumoperitônio deve ser avaliada 
clinicamente duas a três vezes por dia, sendo necessário, por vezes, diminuir ou interromper as insuflações.

Rotineiramente a antibioticoterapia não deve ser utilizada durante a manutenção do pneumoperitônio, sendo indicada, apenas, por ocasião da correção da hérnia, consistindo na infusão de 1 ou $2 \mathrm{~g}$ de cefalozina na indução anestésica e mantida por, no máximo, 24 horas após a cirurgia.

\section{Resultados}

Durante período de 36 meses, seis pacientes com grandes hérnias com perda do domicílio na cavidade abdominal, foram admitidos e operados no Hospital das Clínicas da Faculdade de Medicina de Botucatu - UNESP. Destes seis, quatro pacientes eram do sexo feminino e dois do sexo masculino. A idade mínima foi de 40 e máxima de 62 anos. O mais longo prazo de duração da hérnia era de 40 anos e o menor tempo foi de 5 anos (Tabela 1).

Quatro doentes apresentavam hérnia incisional, sendo que três já tinham sido operados uma vez para correção do defeito. Uma paciente apresentava hérnia umbilical primária desde o nascimento, e um paciente tinha hérnia inguinal primária.

Cinco pacientes apresentavam índice de massa corpórea (IMC) acima de 30, sendo que em dois, este era superior a 40.

Dois pacientes eram hipertensos bem controlados e uma tinha síndrome metabólica múltipla. Dois apresentavam importantes alterações tróficas de pele, com ulcerações.

Todos foram hospitalizados para indução do pneumoperitônio e mantidos durante a instalação, cirurgia e pós-operatório. $\mathrm{O}$ tempo médio de pneumoperitônio foi de 11,6 dias (variação de 10 a 15 dias).

Em todos os pacientes o pneumoperitônio foi realizado no hipocôndrio esquerdo, sendo que em dois o procedimento foi realizado no centro cirúrgico, sendo um sob anestesia geral, porque a paciente tinha síndrome de Down, sem garantias de cooperação. No outro caso, a paciente era obesa mórbida com síndrome metabólica múltipla. Nesses dois casos a punção foi realizada com agulha de Veress, conforme procedimento descrito anteriormente.

Nenhum dos pacientes apresentou complicações relacionadas à punção ou manutenção do pneumoperitônio. Todos apresentaram algum tipo de desconforto durante a manutenção do procedimento, como dor abdominal, em ombros, e saciedade precoce, sem ser necessário encerrar a insuflação. Em quatro casos, foi necessário diminuir o volume de ar insuflado, sendo também utilizado analgésico comum (dipirona).

Somente uma paciente obesa mórbida recebeu antibioticoterapia, desde a introdução do cateter, até 72 horas de pós-operatório, pois apresentava ulcerações de pele e alterações tróficas.
Aproveitou-se da internação para dispensar cuidados locais, como tratamento do intertrigo e das lesões de pele descritas (Figuras 3 e 4 ).

O restante dos casos recebeu apenas uma dose de antibiótico profilático na indução anestésica (cefazolina) e mantido por, no máximo, 24 horas.

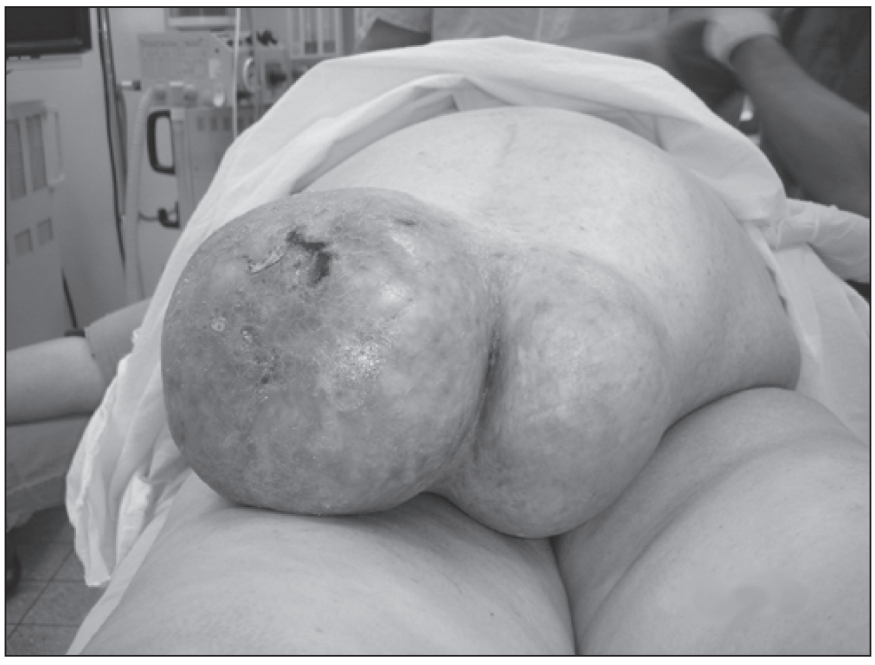

FIGURA 3. Paciente $n^{\circ} 2$. Grande hérnia incisional infraumbilical, com lesões da pele, tratadas por 15 dias, imediatamente antes da cirurgia

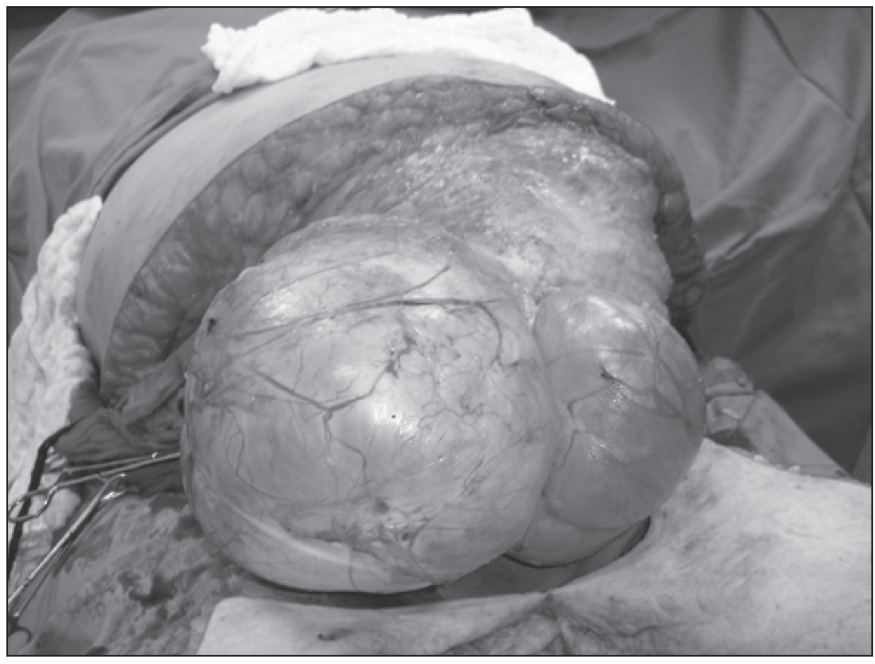

FIGURA 4. Paciente $n^{\circ}$ 2. Observe a dissecação do saco herniário pelo pneumoperitônio

TABELA 1. Localização, origem, tempo de evolução, dias de pneumoperitônio, técnica de hernioplastia

\begin{tabular}{|c|c|c|c|c|c|}
\hline Paciente $\left(\mathrm{n}^{\circ}\right)$ & Local do defeito & Origem da hérnia & Tempo de evolução (anos) & Dias de pneumoperitônio & Técnica hernioplastia \\
\hline 1 & umbilical & primária & 40 & 15 & Tela \\
\hline 2 & infraumbilical & cesariana & 9 & 15 & Tela \\
\hline 3 & infraumbilical & cesariana & 8 & 10 & Tansposição peritônio-aponeurótico \\
\hline 4 & infraumbilical & laparotomia ginecológica & 6 & 10 & Tela \\
\hline 5 & supraumbilical & esplenectomia & 5 & 10 & Tela \\
\hline 6 & inguinal esquerda & primária & 18 & 10 & Lichtenstein \\
\hline
\end{tabular}


Todos os pacientes tiveram a pressão intra-abdominal monitorada no pós-operatório, através de cateter vesical. Os valores se mantiveram normais em todos os casos.

O conteúdo herniário foi colocado de volta à cavidade abdominal sem dificuldades em todos os pacientes.

Quanto aos procedimentos realizados, o paciente com hérnia inguinal foi submetido a herniorrafia, conforme proposto por LICHTENSTEIN $^{(5)}$. Em uma paciente com grande hérnia incisional infraumbilical, utilizou-se a transposição peritônioaponeurótica, conforme proposto por LÁZARO-da-SILVA ${ }^{(4)}$.

As três pacientes que apresentavam hérnias incisionais infraumbilicais e a que apresentava hérnia umbilical gigante, foram submetidas a abdominoplastia associada (Figuras 5 e 6 ). O tempo operatório variou de 150 a 190 minutos. O período de internação pós-operatório variou de 3 a 32 dias. O período de internação maior aconteceu na paciente com síndrome de Down que apresentava grande hérnia umbilical, presente desde o nascimento. A operação tinha ocorrido muito bem, assim como o $1^{\circ}$ e o $2^{\circ}$ dias de pós-operatório, quando apresentou episódio de broncoaspiração e insuficiência respiratória, tendo evoluído para o óbito no $32^{\circ}$ dia de pós-operatório, em decorrência de infecção respiratória e septicemia.

Houve um único caso de infecção da ferida operatória, que ocorreu numa paciente obesa submetida a abdominoplastia que, felizmente, evoluiu bem com tratamento clínico.

Todos os pacientes operados, com exceção daquela que foi a óbito, encontram-se bem, satisfeitos com a intervenção e sem sinais de recidiva, num período de seguimento de 4 a 36 meses.

\section{DISCUSSÃO}

Hérnias crônicas e volumosas da parede abdominal, contendo grande quantidade de vísceras, são extremamente difíceis de se reparar. São consideradas hérnias que perderam o domicílio na cavidade abdominal, haja vista que seu conteúdo excede

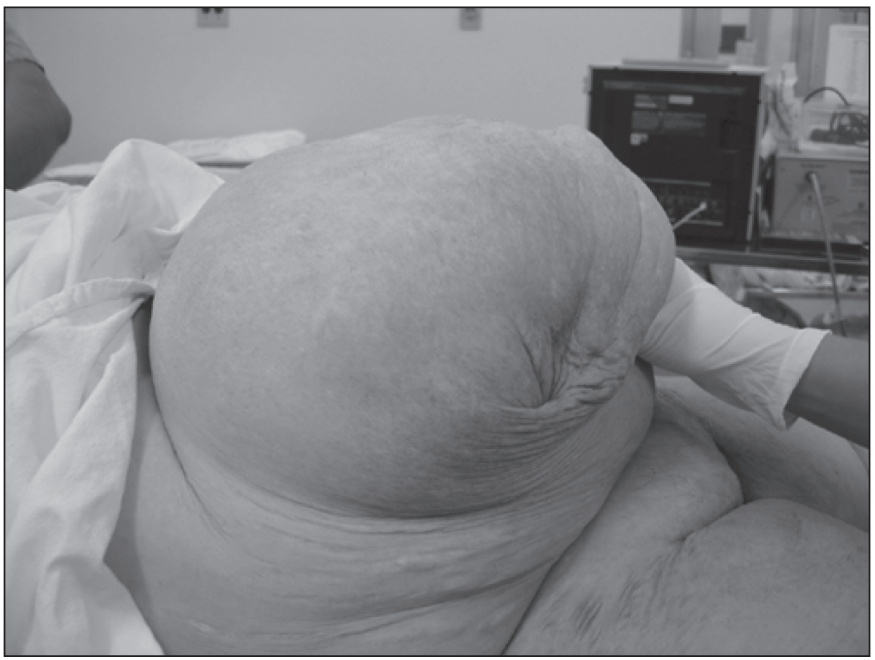

FIGURA 5. Paciente n 1 . Grande hérnia umbilical, imediatamente antes da instalação do pneumoperitônio a sua capacidade. A redução forçada destas hérnias pode levar à insuficiência respiratória, síndrome compartimental abdominal ou mesmo, a impossibilidade de se concluir a hernioplastia ${ }^{(9)}$.

O pneumoperitônio progressivo pré-operatório é possivelmente a melhor conduta a ser adotada nesses casos, pois permite a realização do procedimento com certa tranqüilidade e riscos aceitáveis.

Criado por GOÑI-MORENO ${ }^{(2)}$, na década de 1940, este procedimento foi modificado para facilitação de sua aplicação. Uma das modificações propostas foi substituir o trabalhoso processo de introdução de ar ambiente na cavidade peritonial, utilizando-se um sistema de sifão, que era "lavado" com iodopovidina, para reduzir o risco de peritonite. Basicamente, modificações da técnica original consistiram na maneira de puncionar, no tipo de cateter e na troca do gás utilizado.

CALDIRON et al. ${ }^{(1)}$, em 1990, realizava punções diárias com agulha de Veress e utilizava o $\mathrm{CO}_{2}$. O método tinha o risco de múltiplas punções e da rápida absorção do $\mathrm{CO}_{2}$. MARTINEZ et al. ${ }^{(8)}$ usavam um cateter de duplo lúmen para o pneumoperitônio, sendo o mesmo realizado com ar ambiente não filtrado, sem nenhuma complicação infecciosa.

Embora contaminado, o ar ambiente por si só não é suficiente para produzir peritonite, já que o peritônio possui complexo mecanismo de defesa, que faz com que a simples contaminação, sem substâncias adjuvantes, não leva à infecção ${ }^{(12)}$.

Não se pensou, também, que fosse necessário o uso de antibióticos profiláticos durante a instalação e manutenção do pneumoperitônio, conforme preconizado na literatura médica. Não houve nenhuma complicação com o procedimento na presente casuística. A única paciente que utilizou antimicrobianos, desde a instalação do pneumoperitônio, era obesa mórbida, com síndrome metabólica múltipla e lesões ulceradas de pele à altura do defeito herniário.

Embora o pneumoperitônio progressivo pré-operatório tenha partido de bases científicas, a determinação do volume

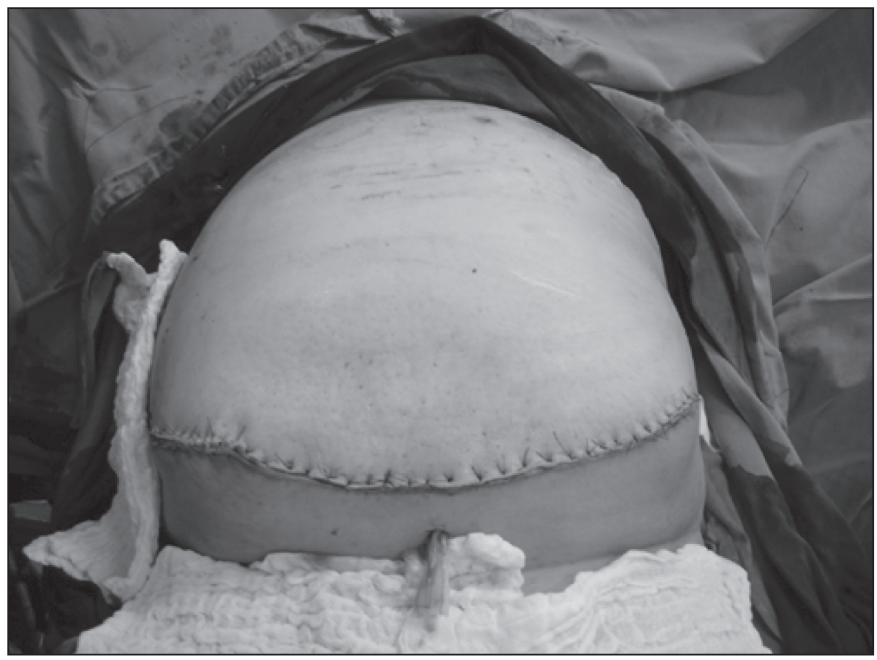

FIGURA 6. Paciente $n^{\circ} 1$ no pós-operatório imediato de cirurgia realizada após 15 dias de pneumoperitônio 
de ar a ser introduzido, por sessão, e o total de aplicações, ainda permanecem cirurgião-dependente, limitando o seu uso generalizado. Acredita-se que período menor que 10 dias e volume abaixo de 10 litros no total infundido, não atingem os objetivos a serem alcançados, que são: elevar novamente a pressão intra-abdominal de forma gradual, estabilizar a forma e a função diafragmática, melhorar a função ventilatória, distender os músculos da parede abdominal, aumentar o volume da cavidade, produzir a lise pneumática das aderências mais frouxas, melhorar a circulação portal, mesentérica e intestinal - para receber de volta as vísceras na cavidade abdominal e produzir irritação peritonial, com vasodilatação e aumento do aporte de leucócitos (macrófagos), que melhoram a resposta cicatricial.

Um dos maiores inconvenientes do pneumoperitônio é a manutenção do paciente internado no pré-operatório, por período longo. Embora o procedimento possa ser realizado em ambiente de ambulatório ${ }^{(9)}$, todos os casos desta série permaneceram internados pois, de modo geral, são pacientes humildes e que residem distante do hospital. Outro aspecto importante é que todos os casos necessitaram de ajustes no volume de ar infundido, medicação analgésica e, às vezes, medicação antiemética. Em nenhum paciente foi necessário interromper o procedimento.

Como é freqüente, os pacientes tiveram longo histórico de hérnias, que variou de 5 a 40 anos. Somente um dos doentes tinha IMC abaixo de 30. Todas as hérnias incisionais tiveram origem em laparotomias medianas.

Com relação às intervenções cirúrgicas, observou-se que em todos os casos, foi possível reduzir completamente os conteúdos dos sacos herniários, o que facilitou muito a confecção da hernioplastia.

Quanto à técnica operatória, pôde-se observar que a maioria dos pacientes necessita do uso de material sintético para a reconstrução da parece abdominal. Mesmo assim, é importante lembrar que nos casos em que o saco herniário é exuberante e resistente, a transposição peritônio-aponeurótico se constitui em técnica atraente, evitando-se as complicações das próteses de modo geral. Embora adeptos da técnica, houve oportunidade de executá-la em um único caso.

Aspecto importante do pós-operatório é o monitoramento da pressão intra-abdominal que deve ser realizado por, pelo menos, 48 horas. Este procedimento pode ser facilmente realizado através de um cateter vesical. Em todos os casos operados, não se observou aumento da pressão intra-abdominal, sinal indicativo da eficiência do pneumoperitônio e ausência da síndrome compartimental.

Outra consideração a ser observada é que a anestesia com óxido nitroso deve ser evitada, pois em geral, não se consegue retirar todo ar imediatamente antes da operação. O gás nitroso entra em equilíbrio com o ar do pneumoperitônio, difundindo-se rapidamente do sangue para a cavidade abdominal, com aumento súbito da pressão abdominal, podendo causar hipovolemia ou insuficiência respiratória. Neste caso, é necessária uma punção abdominal de urgência para esvaziar os gases.

Complicações como o hematoma e o enfisema subcutâneo, embora incomuns, podem ocorrer. $\mathrm{O}$ enfisema mediastinal e o pneumotórax também podem ocorrer, e o fazem por lesão ou delaminação do diafragma, por passagem do ar pela parede do esôfago ou, ainda, pelos hiatos diafragmáticos.

A embolia gasosa é complicação em potencial, e está correlacionada à falha técnica e/ou à injeção de ar no território intra-hepático, intraesplênico ou intravenoso.

$\mathrm{Na}$ presente casuística, não se observaram complicações graves relacionadas ao pneumoperitônio e à intervenção cirúrgica propriamente dita. O óbito ocorrido no pósoperatório, foi decorrente de complicações respiratórias após broncoaspiração, e estava muito mais relacionado às condições desfavoráveis da paciente, do que propriamente em decorrência do procedimento.

Os cinco pacientes restantes encontram-se em seguimento em ambulatório, por período de 4 a 36 meses, referindo estar clinicamente bem e plenamente satisfeitos com o resultado do tratamento.

\section{CONCLUSÃO}

As alterações fisiopatológicas causadas pelas hérnias gigantes da parede abdominal são complexas e podem comprometer o sucesso de uma operação.

O uso do pneumoperitônio progressivo pré-operatório é procedimento relativamente simples e seguro. É indicado em doentes com hérnias que perderam o domicílio na cavidade abdominal.

\section{AGRADECIMENTO}

Ao Prof. Adj. César Tadeu Spadella, pelas revisões do texto e valiosas sugestões. 
Minossi JG, Oliveira WK, Llanos JC, Ielo SM, Hasimoto CN, Pereira RSC. Preoperative progressive pneumoperitoneum in voluminous abdominal wall hernias. Arq Gastroenterol. 2009;46:121-6.

ABSTRACT - Context - Correction of voluminous hernias and large abdominal wall defects is a big challenge in surgical practice due to technical difficulties and the high incidence of respiratory and cardiovascular complications. Objectives - To present the authors experience with inducing progressive pneumoperitoneum preoperative to surgical treatment of voluminous hernias of the abdominal wall. Methods - Retrospective study of six patients who presented voluminous hernias of the abdominal wall and were operated after installation of a pneumoperitoneum. The procedure was performed by placing a catheter in the abdominal cavity at the level of the left hypochondrium with ambient air insufflation for 10 to 15 days. Results - Four of the six patients were female and two male. Ages ranged from 42 to 62 years. Hernia duration varied from 5 to 40 years. Four patients had incisional, one umbilical, and one inguinal hernias. Mean pneumoperitoneum time was 11.6 days. There were no complications related to pneumoperitoneum installation and maintenance. All hernias were corrected without technical difficulties. The Lichtenstein technique was used to correct the inguinal hernia, peritoneal aponeurotic transposition for one of the incisional hernias, with the rest corrected using polypropylene mesh. One death and one wall infection were observed post operatively. No recurrences were reported until now, in 4 to 36 months of follow-up. Conclusion - Preoperative progressive pneumoperitoneum is a safe and easy executed procedure, which simplifies surgery and reduces post-operative respiratory and cardiovascular complications. It is indicated for patients with hernias that have lost the right of domain in the abdominal cavity.

HEADINGS - Pneumoperitoneum, artificial. Hernia, ventral. Hernia, inguinal

\section{REFERÊNCIAS}

1. Caldiron MW, Romano M, Bozza F. Progressive pneumoperitoneum in the management of giant incisional hernias: a study of 41 patientes. Br J Surg. 1990; 77:306-8.

2. Goñi-Moreno I. Chronic eventrations and large hernias: preoperative treatment by progressive pneumoperitoneum - original procedure. Surgery. 1947;22:945-53.

3. Herszage L. Abordaje de las eventraciones de pared. In: Mayagoitia GJC, editor. Hérnias de la pared abdominal: tratamiento actual. México: McGraw-Hill; 2004

4. Lázaro-da-Silva A. Surgical correction of longitudinal median of paramedian incisional hérnia. Surg Gynecol Obstet. 1979;148:579-83.

5. Lichtenstein IL. Herniorraphy. A personal experience with 6,321 cases. Am Surg. 1987;153:553-9.

6. Lima MJV, Silva AL. Pneumoperitônio. In: Lázaro-da-Silva A, editor. Hérnias. São Paulo: Rocca; 2006. p.171-4.

7. Martinez MA. Neumoperitonio en hernias gigantes. In: Mayagoitia G J C, editor. Hernias de la pared abdominal: tratamiento actual. México: Mc Graw-Hll; 2004.
8. Martinez MA, Quijano OF, Padilha LR, Hesiquio SR, Alvarez CO, Medina RO Catéter de doble luz para pneumoperioteno en hernias gigantes. Cir Gen. 2002;24 313-8.

9. Mayagoitia GJC, Suarez FD, Arenas RJC, Dias de Leon. Preoperative progressive pneumoperitoneum in patients with abdominal-wall hernias. Hernia. 2006; 10:213-7.

10. Mayagoitia GJC. Pneumoperitônio progressivo pré-operatório. In: Melo R M, Abdalla R Z, editors. Hérnias da parede abdominal. São Paulo: Iatria; 2007. p. 146-68.

11. Munegato G, Grigoletto R, Brandolese R. Respiratory mechanics in abdominal compartment syndrome and large incisional hernias of the abdominal wall. Hernia. 2000;4:282-5.

12. Rasslan S, Saad Jr R. Peritonites. In: Rasslan S, editor. Afecções cirúrgicas de urgência. São Paulo: Robe; 1995. p.25-46. 